\title{
Measuring the Economy in the Digital Age
}

\author{
Samantha Sanders \\ (Department of Economics, Shanghai University, China)
}

\begin{abstract}
When GDP was created it was considered one of the greatest inventions of its time. Whilst it has always garnered some degree of criticism, the rapid emergence of new technologies in recent years has only increased skepticism towards this economic tool. In particular, the 'sharing economy', as well as the relatively wide-range of free goods and services that are available has raised questions about the accurateness of GDP. Ultimately, there is a strong argument in favor of re-addressing how this tool is compiled and how it is used.

Keywords: digitalization, free products, GDP, globalization, sharing economy
\end{abstract}

\section{Introduction}

Once described as one of the greatest inventions of its time by the US Bureau of Economic Analysis, GDP has come under increasing scrutiny. It's a complicated concept with the end goal of providing a rough estimate of economic progress. It is discussed and dissected by banks and businesses. It is referred to by politicians. Newscasters work to provide updates. And yet, it has always had its critics. After all, GDP does not differentiate between purchases that are "good" or "bad" for the buyer, the value of goods that are provided by nature, or the quality of the goods provided. Robert F. Kennedy, in a speech in 1968, famously stated, "It measures everything in short, except that which makes life worthwhile." [1] There is growing concern that GDP, which was devised in a more industrial era, is less suited for an economy that is both service oriented and digitally driven. The economy that people experience is largely absent from GDP. In 2014 alone, it's estimated that the calculated GDP failed to include around \$1.5 trillion in investments in "intangible" goods, such as software and brand equity. In another example, Japan's economy was said to have contracted by $0.9 \%$ in 2014 . However, an alternate assessment by the Bank of Japan suggests that the economy had actually expanded by 2.4\%. [2] [3] One commonly cited point is the growing phenomenon of the 'sharing economy', which describes the use of online platforms that bring suppliers and consumers together. This trend includes companies such as Airbnb and Uber. Another important aspect is the relatively wide-range of "free" products and services. Consumers can use dictionaries, download apps, play games, watch videos and use software, all for free. These services may not be genuinely "free", but their true value is hard to judge and include in GDP. This is especially true of non-monetary benefits. Adding to the complexity is the globalized nature of many of these products and services, which makes it difficult to assign value or production to any one nation. Ultimately, there is a strong argument in favor of re-examining how GDP is compiled and even what it used for.

\section{The Sharing Economy}

One important aspect of the digital market is that of the 'sharing economy'. The 'sharing economy' refers to the use of online platforms to share underused assets. Google's chief economist, Hal Varian, has argued that GDP cannot truly capture the sharing economy as the consumer benefit is a combination of both standard gains and efficiency gains.[4] The most commonly cited examples of the sharing economy are Uber and Airbnb. In just one year, between 2014 and 2015, Airbnb's market capitalization doubled and surpassed that of Marriot Hotels. The concept of peer-to-peer transactions is not necessarily a revolutionary one. What has changed, however, is the scale of this kind of exchange, which has been largely driven by the fast adoption of online platforms that reduce barriers to entry and increase market size. The question then arises as to whether compilation methods are able to accurately account for this type of transaction at this scale.

In the case of rentals, individuals are required to report the related income to the government for taxation purposes, but there is likely to be a degree of under-reporting associated with short term lets. Before the advent of Airbnb and other similar services, this kind of transaction was relatively small-scale. The value of under-reported, short-term Airbnb lettings are covered, to some degree, by the inclusion of owner-occupied rent in GDP calculations. However, due to the difference in value between short-term and long-term rental values, some value is not reflected. There is not currently enough evidence to ascertain, either way, what the value of that difference is. Outside of under-reporting, there is also the issue of services that are provided for free. Couchsurfing, for example, involves hosts offering travelers a free play to sleep. Lastly, there are the benefits that are even more difficult to capture, such as the positive environmental impact that may arise from the use of these kinds of services. Fortunately, the rise of the use of registered intermediaries also means that these transactions are being recorded. For authorities this means that there is a means by which they can create estimates of any additional value that is being overlooked. [5] 


\section{III. "Free" Goods and Services}

The wide-range of available "free" products and services is also often cited as activity that GDP fails to account for. After all, how does one measure something with no price and no sales revenue?Consumers can download apps, search the internet, participate in online social networks, access open source software and watch videos, all for free. The scale of this shouldn't be underestimated. Pokémon Go, for instance, became a sensation overnight. The game was available to download for free, and yet it led to overnight gains by Nintendo. [6] Furthermore, free services and products make it difficult to truly measure how well-off citizens are. Dictionaries and encyclopedias are goods that were previously provided at a cost, but are now available for free. Households are clearly better off, but as they are accessing "free" products, this change may not be well-reflected.

The market of free services has also given rise to an increasingly common situation wherein consumers take on roles that were previously managed by a dedicated intermediary. For example, flight bookings used to be largely handled by travel agents, but they are now often booked by the consumer directly via travel websites. These kinds of services have transformed the way that consumers interact with businesses. [7] In the case of some free services, such as Wikipedia, there is the added complication of cross-border activity. Wikipedia is global in nature, and it is therefore difficult to assign the assets to any particular nation.

It is possible that revenues from free goods are simply being redistributed. It is often true, after all, that nothing is ever really free. Consumers have long received free products from businesses as a method of advertising. In this model, companies typically pay advertising companies for a service and then, presumably, recoup the loss via the additional revenue that the advertising campaign attracts. Today's "free" product market may just be a more complex version of this setup. Consumers using the free app or online service are paying service providers for broadband. Advertisers are paying the app or website to allow them to display adds. The free app or online service itself is earning money by selling data about their users. As a another example, data suggests that while jobs at traditional newspaper companies has dropped, employment at smaller, new media firms has ticked up. The crux of the problem is that there simply isn't enough data to make a judgment on the situation. [8]

\section{Conclusion}

GDP has garnered criticism since its conception. Yet, it has remained as the frontline tool for measuring economic progress for well over half a century. It worked well enough when the economy produced things that were easily countable. Now, however, the wave of criticism is intensifying. The economy is in the midst of a transformation so substantial that GDP is viewed an increasingly ill-suited tool. The rise in trends such as the 'sharing economy' and the wave of "free" goods have created value that is difficult to measure. This is especially true in regards to quality and overall consumer benefit. If, for example, an airline squeezes more customers onto a plane, this is counted as additional output, even if quality has dropped. [9]

There is, however, no consensus about what changes should be made, or even whether GDP should be continued at all. After all, it seems unusual in many ways to rely so strongly on a single metric. There is also some question as to the purpose of GDP. Is it largely a tool for measuring economic welfare? Is it, alternatively, a purely macroeconomic tool? In any case, it is alarming when one considers how much attention is paid to this rather fallible figure. For this reason alone it is worth having a serious, collective discussion about GDP, both in terms of how it's composed and how it's used.

\section{References}

[1]. R.F. Kennedy, Remarks at the University of Kansas, Speech, The University of Kansas, Kansas, March 18, 1968.

[2]. D. Coyle, Why GDP Statistics Are Failing Us, U.S. Chamber of Commerce Foundation.

[3]. P. Wallace, Commentary: The GDP is a grossly defective product. We need to knock it off its pedestal, Reuters, October $20,2016$.

[4]. D. Coyle,Why GDP Statistics Are Failing Us, U.S. Chamber of Commerce Foundation.

[5]. N. Ahmad, P. Schreyer, Measuring GDP in a digitized economy,OECD Statistics Directorate, IMF, 2016.

[6]. C. Sorensen, GDP is actually a pretty terrible way to measure economic growth, Canadian Business, August 18, 2016.

[7]. N. Ahmad, P. Schreyer, Measuring GDP in a digitized economy, OECD Statistics Directorate, IMF, 2016.

[8]. D. Coyle, Why GDP Statistics Are Failing Us, U.S. Chamber of Commerce Foundation.

[9]. J.O.S., S., Why GDP is a poor measure of progress, The Economist. May 09, 2016. 